\title{
Brachionidae (Rotifera: Monogononta) de la albufera El Paraíso y el reporte de Brachionus ibericus en el Perú
}

\author{
Brachionidae (Rotifera: Monogononta) from El Paraíso albufera and report of Brachionus ibericus \\ in Peru
}

\author{
Emily Toscano y Ruperto Severino
}

Laboratorio de Protozoología e Invertebrados acelomados y pseudocelomados, Departamento de Zoología, Facultad de Ciencias Biológicas, Universidad Nacional Mayor de San Marcos. Apartado postal 11-0058, Lima, 11, Perú.

Email Emily Toscano: ema.tg27@gmail.com

\section{Citación:}

Toscano E. \& R. Severino. 2013. Brachionidae (Rotifera: Monogononta) de la albufera El Paraíso y el reporte de Brachionus ibericus en el Perú. Rev. peru. biol. 20(2): 177 - 180 (Diciembre 2013)

\section{Resumen}

En este trabajo se reportan las especies de Brachionidae reconocidos en las muestras recogidas en octubre de 2010 en la albufera Laguna El Paraiso, Huacho $\left(11^{\circ} 13^{\prime}-11^{\circ} 10^{\prime} \mathrm{S}, 77^{\circ}\right.$ $35^{\prime}-77^{\circ} 40^{\prime} \mathrm{W}$ ). Cinco especies fueron determinadas: Keratella tropica, Brachionus quadridentatus, B. urceolaris, B. angularis y B. ibericus Ciros-Pérez 2001. Este último es un nuevo reporte para el Perú y amplía su distribución a nivel mundial.

Palabras clave: Zooplancton; especie cosmopolita; especie críptica; complejo Brachionus plicatilis.

\section{Abstract}

This paper reports the Brachionidae species recognized in samples collected in October 2010 in the albufera Laguna El Paraiso, Huacho $\left(11^{\circ} 13^{\prime}-11^{\circ} 10^{\prime} \mathrm{S}, 77^{\circ} 35^{\prime}-77^{\circ} 40^{\prime} \mathrm{W}\right)$. Five species were determined: Keratella tropica, Brachionus quadridentatus, $B$. urceolaris, B. angularis and B. ibericus Ciros-Pérez 2001, which is a new report for Peru and this report expanding its worldwide distribution.

Keywords: Zooplankton; cosmopolitan species; cryptic specie; Brachionus plicatilis complex.

\section{Introducción}

Los Rotifera son componentes del zooplancton y especies importantes en las cadenas tróficas de los ecosistemas de aguas continentales. Su distribución geográfica es muy amplia, pueden encontrarse desde el mar hasta charcos estacionales de agua (Wallace 2002). Se han descrito más de de 2000 especies de rotíferos y la mayoría con tamaños entre $100-1000 \mu \mathrm{m}$ de longitud. La región Neotropical, es la tercera más diversa, presentando 566 especies de la clase Monogononta, destacando la familia Brachionidae con 71 especies (Brachionus con 32 y Keratella con 18) y 116 especies de la clase Bdelloidea (Segers 2008).

Dentro del phylum Rotifera, el género Brachionus inluye el 80\% de las especies del phylum (Segers 2007), y es conocido como "el ratón blanco" de los rotíferos, siendo objeto de múltiples estudios, desde ecoevolutivos hasta bioquímicos, y utilizado como modelo en estudios fisiológicos y toxicológicos (Gallardo et al. 2006, Lee et al. 2010, Ciros-Pérez et al. 2001)

Sin embargo, el estudio de Brachionus ha tenido dificultades debido a la variación fenotípica que presentan algunas especies (Yin \& Niu 2007), así como a la ocurrencia de especies simpátricas morfológicamente similares pero con requerimientos distintos, conformando grupos de especies crípticas, tales como el complejo Brachionus plicatilis (Ciros-Pérez et al. 2001) afectando de esta manera el conocimiento de su verdadera distribución geográfica (Jersabek \& Bolortsetseg 2010).

En el Perú, se conoce poco acerca de la diversidad y distribución los rotíferos. Los primeros reportes en el Perú, acerca de la composición y morfología de los rotíferos fueron dados a conocer por Samanez $(1988,1991)$, y por Samanez \& Riofrio (1995) en estudios realizados en la Amazonía peruana. En humedales del departamento de $\begin{array}{ll}\text { Presentado: } & 09 / 01 / 2013 \\ \text { Aceptado: } & 08 / 09 / 2013\end{array}$

$\begin{array}{ll}\text { Aceptado: } & 08 / 09 / 2013 \\ \text { Publicado online: } & 09 / 12 / 2013\end{array}$ 

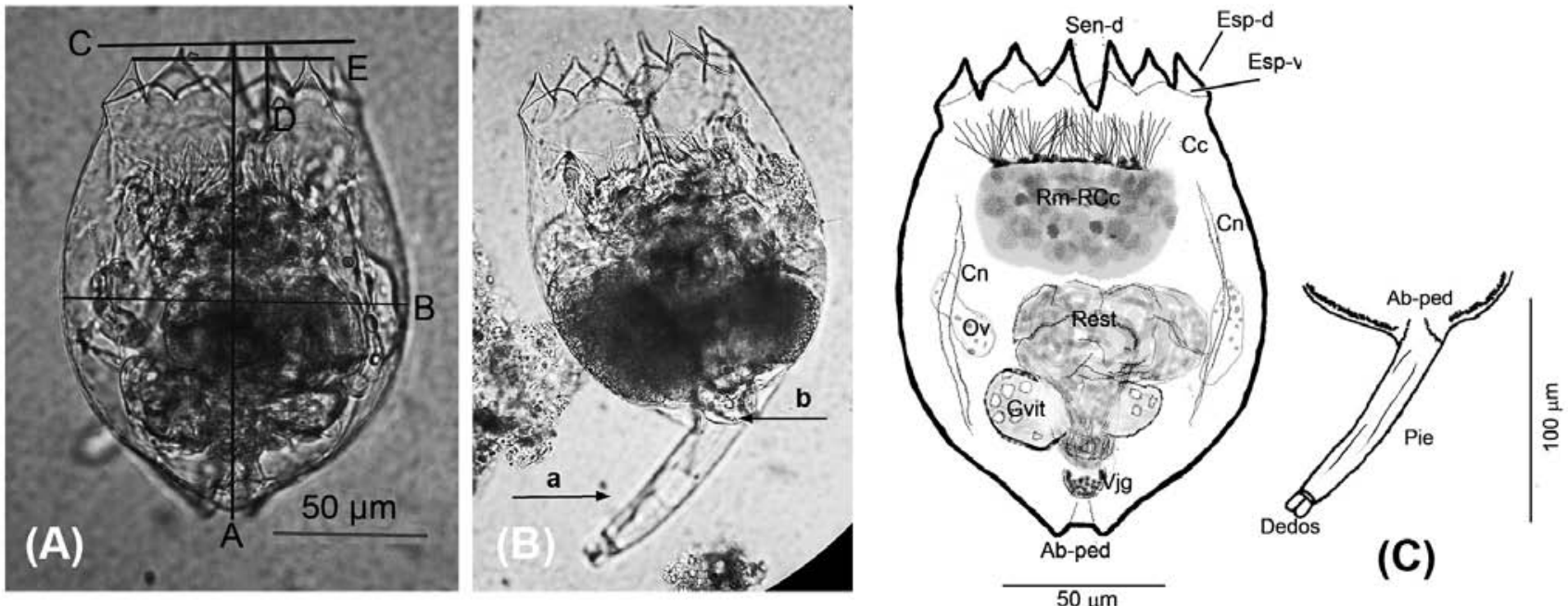

Figura 1. Brachionus ibericus. (A) Mediciones de la Lórica. A: Longitud Lórica. B: Ancho Lórica. C: Apertura Cefálica. D: Profundidad de espina central y E: Distancia interespinal (B) Detalle de a) pie elongado, que sale a partir de b)apertura pedial. Aumento: 40X. (C) Esquema de las estructuras de B. ibericus Ab-ped: Abertura pedial. Cc: Corona Ciliar. Cn: Restos de Cordón nervioso. Esp-d: Espina dorsal. Esp-v: Espina ventral. Gvit: Glándula vitelina. Rm: Restos del mástax. RCc: Restos de la Corona ciliar. Rest: Restos estomacales. Sen-d: Seno ventral. Vjg: Vejiga

Lima se han reportado rotíferos en trabajos de índole ecológico y prospectivo (Iannacone \& Alvarińo 2007, Paredes et al. 2007).

La albufera o laguna El Paraíso es un humedal costero ubicado en el distrito de Huacho, aproximadamente $145 \mathrm{~km}$ de Lima $\left(11^{\circ} 13^{\prime}-11^{\circ} 10^{\prime} \mathrm{S}, 77^{\circ} 35^{\prime}-77^{\circ} 40^{\prime} \mathrm{W}\right)$. El Paraíso forma parte de un conjunto de humedales a lo largo de la costa peruana y representa un refugio para una gran diversidad de aves, de las cuales el $43,2 \%$ son migratorias continentales y locales (Cruz et al. 2007).

Las colectas de muestras se realizaron en octubre de 2010 en tres puntos del espejo de agua principal, en la superficie del agua y a un metro de orillas con profundidades de más de un metro. Se uso una red de fitoplancton $(40 \mu \mathrm{m})$ con dos procedimientos, uno realizando un arrastre superficial en forma circular, y un segundo un arrastre vertical desde el fondo a la superficie. Las muestras fueron colocadas en frascos de $100 \mathrm{~mL}$, y fijadas con formol en una concentración final de 4\%. Los análisis se realizaron con un microscopio óptico a 400 aumentos y una cámara digital Olympus de $12 \mathrm{Mpx}$. Las claves utilizadas fueron de Grothe y Grothe (1977) y Fontaneto (2008).

Se determinó la presencia de una especie de Keratella, cuatro especies de Brachionus y una especie críptica, es decir, cuya morfología es similar a $B$. plicatilis.

Las cuatro especies de Brachionus corresponden a B. angularis, B. quadridentatus, B. ibericus y B. urceolaris. Y el género Keratella está representada por $K$. tropica.

Brachionus ibericus Ciros-Pérez fue descrita en el 2001 y su distribución hasta hoy se creía que estaba restringida a Europa y Asia, exactamente en la ecoregión Paleartica occidental y oriental (Jersabek \& Bolortsetseg 2010). Nuestros resultados permiten constatar la presencia de $B$. ibericus, lo que significa un nuevo reporte para el Perú y para América, ampliando su distribución en el mundo. Nuestras observaciones concuerdan con la descripción de Ciros-Pérez et al. (2001) y Jersabek \& Bolortsetseg (2010), y también son concordantes en relación a las magnitudes que se mencionan en la Tabla 1 (Fig. 1).

\section{Taxonomía}

Descripción de las especies de acuerdo a Ciros-Pérez et al. (2001) y Jersabek \& Bolortsetseg (2010):

$\begin{array}{ll}\text { Phylum: } & \text { Rotifera } \\ \text { Clase: } & \text { Monogononta } \\ \text { Orden: } & \text { Ploima } \\ \text { Familia: } & \text { Brachionidae }\end{array}$

\section{Brachionus ibericus Ciros-Pérez 2001}

Recolectado a nivel superficial y a profundidad. Es euplanctónico de aguas atalásicas, estanques y lagos ausalinos, presente por temporadas o permanentemente, prefiriendo temperaturas mayores a los $15^{\circ} \mathrm{C}$. Su lórica es relativamete suave, lisa y flexible, de forma ovoidea, apertura cefálica medio ancha. Margen dorsal con seis espinas triangulares de tamaños similares, tres a cada lado de un seno en forma de V. Las espinas internas más prominentes, y las externas menos desarrolladas. Las espinas medias en forma de triángulo equilátero. El margen ventral anterior con dos pares de lóbulos flanqueando un seno estrecho a la altura del seno dorsal. Apertura pedial subterminal, sobre una placa ventral (Figs. 1A y B).

Tabla 1. Medidas promedios $(\mu \mathrm{m})$ para Brachionus ibericus según Ciros-Pérez et al. 2001

\begin{tabular}{lcc}
\hline MEDIDAS & $\begin{array}{c}\text { Ciros-Pérez et } \\
\text { al. 2001 }\end{array}$ & $\begin{array}{c}\text { Presente } \\
\text { trabajo }\end{array}$ \\
\hline Longitud Lórica (LL) & $193.5 \pm 2.5$ & 170.8 \\
Ancho Lórica (AL) & $144.5 \pm 2.5$ & 124.5 \\
Apertura Cefálica (AC) & $99.0 \pm 2.5$ & 93.0 \\
Profundidad de espinas central & $21 \pm 1$ & 22.1 \\
*LL/AC= & 1.95 & 1.84 \\
*AL/AC= & 1.46 & 1.34 \\
${ }^{*}$ LL/AL= & 1.34 & 1.37 \\
\hline
\end{tabular}

*Relaciones entre las magnitudes dadas. 

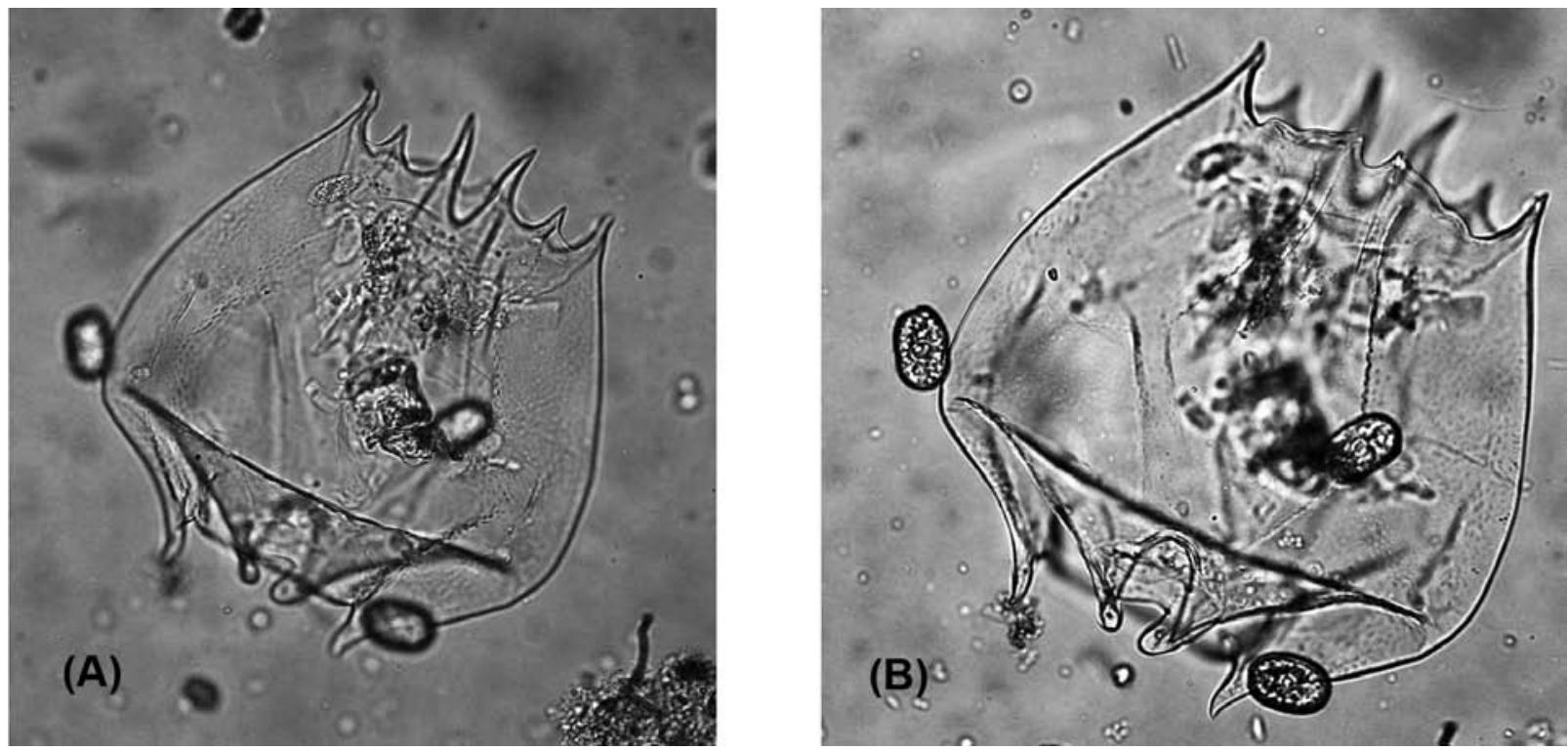

Figura 2. Brachionus cuadridentatus. (A) Vista dorsal, 6 espinas dorsales, espina centrales prominentes. (B) Vista ventral, detalle el margen ventral de la lórica con el seno medio muy estrecho. Aumento: 40X

\section{Brachionus quadridentatus Herman 1783}

Recolectado a nivel superficial. Es una especie bentónica y semiplanctonica entre vegetación acuática en lagunas de agua dulce, ríos, embalses y lagos poco profundos, entre perifiton y macrófitas, también de aguas costeras salobres, de hábitat temporal o permanente, en pantano, aguas eurihalina. Lorica con la placa dorsal y ventral moderadamente aplanado dorsoventralmente, por lo general punteado o pustulante. Margen Antero-dorsal con seis espinas, espinas medianas generalmente más largas, curvadas, granuladas o aserradas. Especie altamente variable debido a las espeinas postero-laterales (Figs. 2A y B).

\section{Brachionus angularis Gosse 1851}

Recolectado a nivel superficial y a profundidad. Es una especie cosmopolita, euplanctónica en lagos de agua dulce, lagunas, estanques y embalses; ocasionalmente en aguas salobres y aguas salinas atalásicas. Lórica con la placa dorsal y ventral

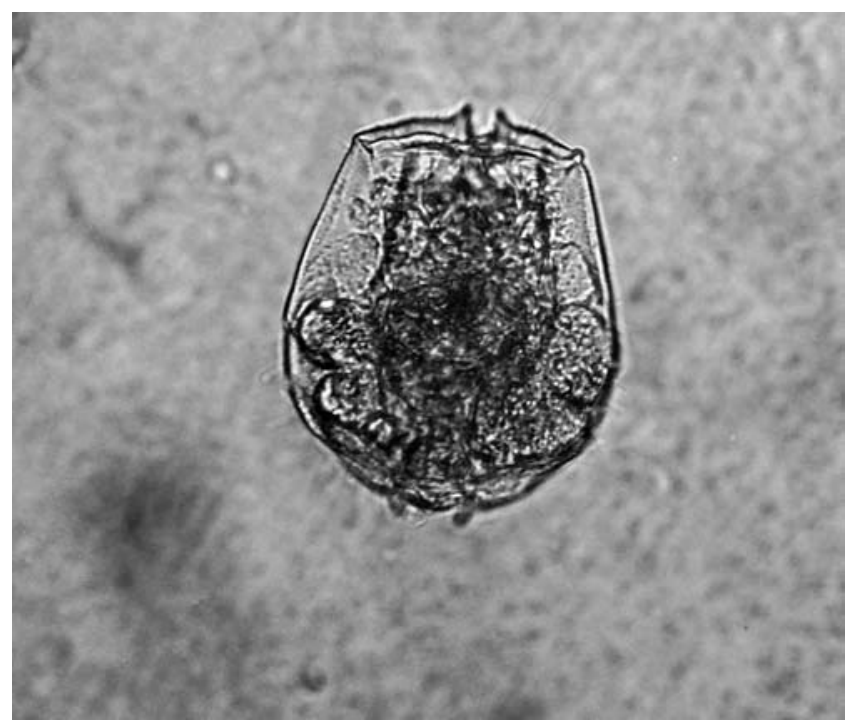

Figura 3. Brachionus angularis Vista ventral. Aumento 40X moderadamente aplanado dorso-ventral y firme. Placa dorsal con un fuerte o muy débil patrón de crestas. Dos espinas medianas cortas antero-dorsales; espinas intermedias y laterales ausentes o fuertemente reducidas, espinas posteriores ausentes (Fig. 3).

\section{Brachionus urceolaris Muller 1773}

Punto A superficial. Es cosmopolita, presente en las ecorregiones Antártida y Pacifico. Bentónicos y semiplanctonicos, predominantemente entre perifiton de macrófitos de agua dulce, estanques, ríos, embalses, lagos, llanuras aluviales inundadas, también en aguas costeras de agua salobre y atalásicas. Temporales o permanentes, euritermicos, eurihalinos. El cuerpo es aplanado dorso-ventralmente. La lorica es suave, firme y se divide en una placa dorsal y ventral que están completamente fusionadas lateralmente. Es ovalada, sin placa basal. En el margen dorsal anterior seis espinas puntiagudas son visibles. Las medianas son más largas que las laterales (Fig. 4).

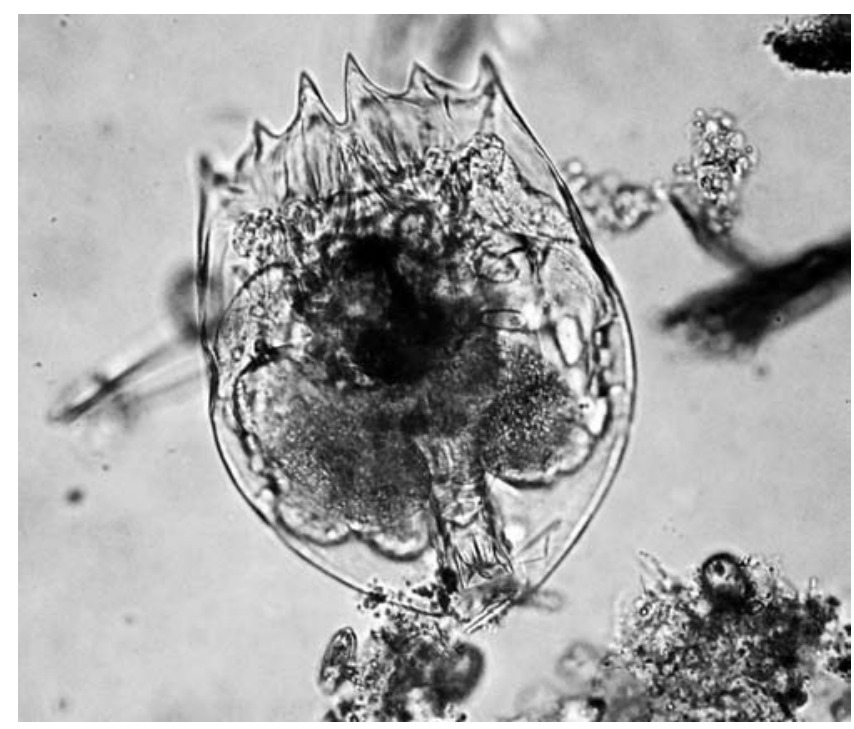

Figura 4. Brachionus urceolaris Vista dorsal. 

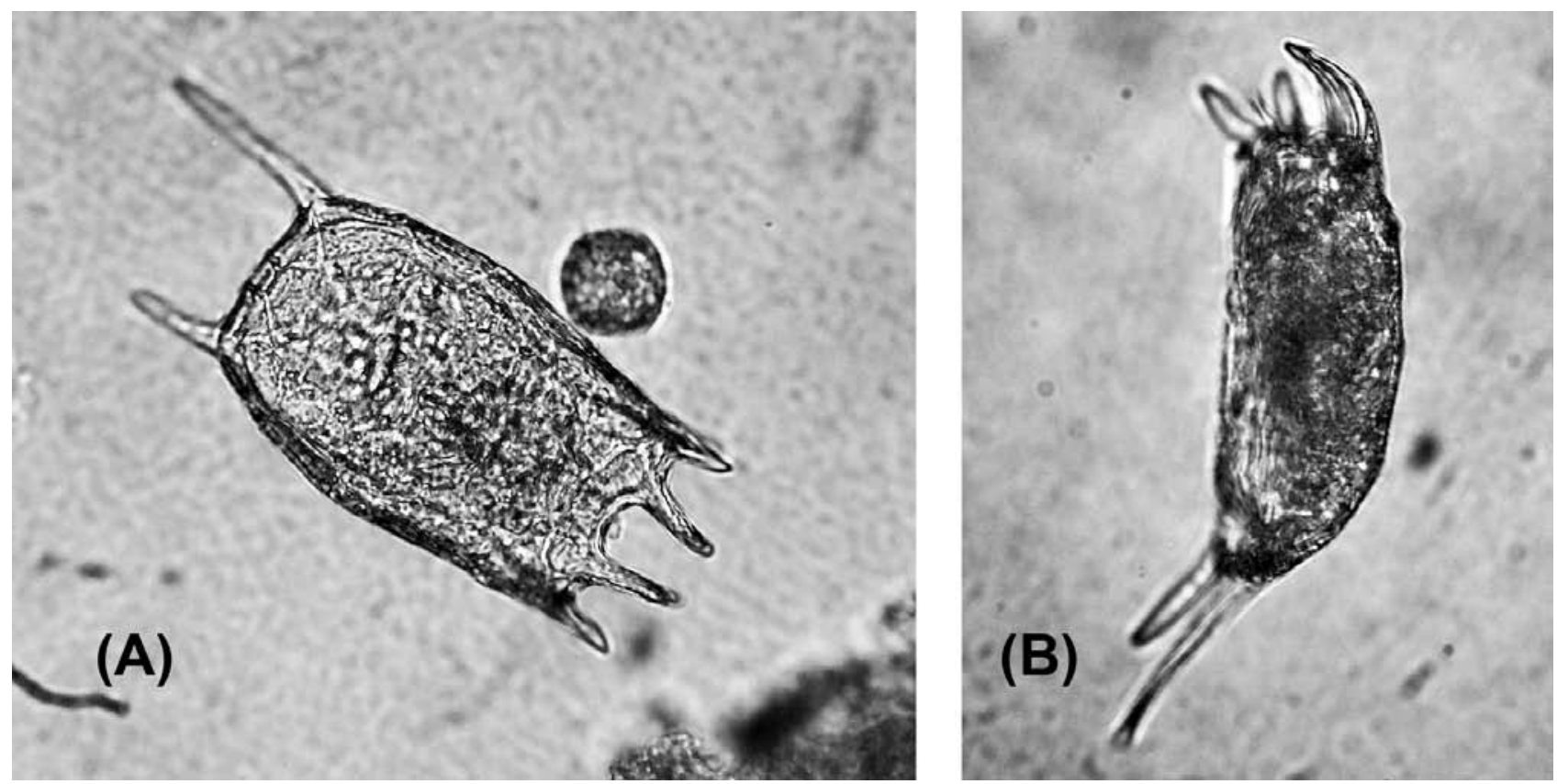

Figura 5. Keratella tropica, (A) Vista dorsal, Lorica con estrías. (B) Vista lateral. Aumento: 40X

\section{Keratella tropica (Apstein 1907)}

Recolectado en profundidad. Es una especie que se encuentra ampliamente distribuida en aguas tropicales y se extiende con mucha frecuencia en las zonas subtropicales, con clima en forma de verano. Su forma típica lleva dos espinas posteriores en la lorica ornamentada, de las cuales la espina izquierda es más corta que la derecha (Kaya \& Altinding 2007) (Fig. 5).

Aunque el trabajo se realizó en un día de muestreo, las especies colectadas amplían el conocimiento de la diversidad de rotíferos, importante para el manejo cepas útiles en acuicultura que beneficie la producción acuícola así como para su uso como fuentes de investigación en campos como la ecotoxicología y ecoevolución.

\section{Agradecimientos}

Nuestro agradecimiento al Dr. Christian Jersabek del Dept. of Organismal Biology, University of Salzburg, por su ayuda en la identificación de B. ibericus. A Ricardo Ricce Bazán por su colaboración en la recolección de muestras y a Alberto López Sotomayor por sus recomendaciones para la realización de este trabajo.

\section{Literatura citada}

Ciros-Pérez J., Gómez A. \& Serra M. 2001. On the taxonomy of three sympatric sibling species of the Brachionus plicatilis (Rotifera) complex from Spain, with the description of B. ibericus n. sp. Journal of Plankton Research 23(12): 1311-1328.

Cruz Z., F. Angulo, H. Burger \& R. Borgesa. 2007. Evaluación de aves en la Laguna el Paraíso, Lima, Perú. Rev. peru. biol. 14(1): 139-144.

Fontaneto. 2008. Identification Key to the Genera of Marine Rotifers Worldwide. Meiofauna Marina 16 (1): 75-99

Gallardo W.G., A. Hagiwara, K. Hara \& K. Soyano. 2006. Growth hormonelike substance in the rotifer Brachionus plicatilis. Fisheries Science 72(4): 781-786.
Grothe D.W. \& D.R. Grothe. 1977. An Illustrated Key to the Planktonic Rotifers of the Laurentian Great Lakes. 1st ed. Vol. 1. U.S. Environmental Protection Agency, Region V, Central Regional Laboratory.

Iannacone J. \& L. Alvariño 2007. Diversidad y abundancia de comunidades zooplanctonicas litorales del humedal Pantanos de Villa, Lima, Perú. Gayana 71(1): 49-65.

Jersabek C.D. \& E. Bolortsetseg. 2010. Mongolian rotifers (Rotifera, Monogononta) - a checklist with annotations on global distribution and autecology. Proc. Acad. Nat. Sci. Philadelphia 159(1): 119-168.

Kaya M. \& A. Altindag. 2007. Brachionidae (Rotifera: Monogononta) Species from Turkey. Asian Journal of Animal Sciences 1 (1): 40-47. doi:10.3923/ajas.2007.40.47.

Lee J.K., M.S. Lee, H.G. Park, S.K. Kim \& H.G. Byun. 2010. Angiotensin I converting enzyme inhibitory peptide extracted from freshwater zooplankton. J. Med. Food. 13(2):357-363.

Paredes C., J. Iannacone \& L. Alvarińo. 2007. Biodiversidad de invertebrados de los humedales de Puerto Viejo, Lima. Perú. Neotrop. Helminthol 1(2): 21-30.

Samanez I. 1988. Rotíferos planctónicos de la Amazonía peruana I: Departamento de Ucayali, Perú. Rev. peru. Biol. 3(1): 141-167.

Samanez I. 1991. Rotíferos planctónicos de la Amazonía Peruana II: Departamento de Loreto. Publicaciones De Museo de Historia Natural 38: $1-4$.

Samanez I. \& Riofrio C. 1995. Composición de la fauna de Rotíferos y su relación con las macrofitas acuáticas en una laguna fluvial, Ucayali. Publicaciones del Museo de Historia Natural 50: 20-30.

Segers H. 2007. Annotated checklist of the rotifers (Phylum Rotifera), with notes on nomenclature, taxonomy and distribution. Zootaxa 1564: 1-104.

Segers H. 2008. Global diversity of rotifers (Rotifera) in freshwater. In: E.V. Balian, C. Lévêque, H. Segers \& K. Martens, (eds.) Freshwater Animal Diversity Assessment. Developments in Hydrobiology. Springer Netherlands. Pp. 49-59.

Wallace R. L. 2002. Rotifers: exquisite Metazoans. Integrative and Comparative Biology 42(3): 660-667.

Yin X. W. \& Niu C.J. 2007. Polymorphism and morphotype transformations in the Rotifer (Brachionus calyciflorus). Zoological Research 28(1): 68-72. 